\title{
Elastic and Transport Properties in Polycrystals of Cracked Grains: Cross-Property Relations and Microstructure
}

\author{
James G. Berryman ${ }^{1, *}$ \\ ${ }^{1}$ University of California, Lawrence Berkeley National Laboratory, \\ 1 Cyclotron Road MS 90R1116, Berkeley, CA 94740, USA
}

\begin{abstract}
Some arguments of Bristow (1960) concerning the effects of cracks on elastic and transport (i.e., electrical or thermal conduction) properties of cold-worked metals are reexamined. The discussion is posed in terms of a modern understanding of bounds and estimates for physical properties of polycrystals - in contrast to Bristow's approach using simple mixture theory. One type of specialized result emphasized here is the cross-property estimates and bounds that can be obtained using the methods presented. Our results ultimately agree with those of Bristow, i.e., confirming that microcracking is not likely to be the main cause of the observed elastic behavior of cold-worked metals. However, it also becomes clear that the mixture theory approach to the analysis is too simple and that crack-crack interactions are necessary for proper quantitative study of Bristow's problem.
\end{abstract}

*JGBerryman@LBL. GOV 


\section{Introduction}

Since some physical properties are easier to measure than others — for example, electrical conductivity versus elastic constants - it is of very practical concern whether or not it might be possible to learn something useful about some hard-to-measure quantity from an easierto-measure one. These considerations lead naturally to the concept of cross-property bounds and estimates [1-7]. Such ideas were applied very early to cold-worked metals by Bristow [8] in order to determine whether or not micro-cracking was an important issue. Bristow's work was so early that modern methods of analysis such as the Hashin-Shtrikman bounds $[9,10]$ had not yet been invented. Bristow [8] treated the microstructure of metals in a very simple way by assuming that cracks were distributed uniformly and isotropically in space, using what we will term "mixture theory." But metals in crystalline form typically have anisotropic elastic response to deformation, and in particular the cold-worked metals that he was considering could perhaps be better thought of as polycrystals, and thereby take the local crystalline behavior into account in the analysis. So the main purpose of the present work is therefore to reexamine some of the technical issues within Bristow's work, and then combine these ideas with modern bounding methods for physical properties. One particularly important point that will be made here is that the microstructure of the

composite material matters a great deal - especially when the constituents are anisotropic. And, if we know even a little bit about this microstructure, this information can improve the precision of our estimation capability — as shall be demonstrated.

For metals, we have in mind a two-stage process of homogenization: (a) The first step will involve a pure metal in polycrystalline - and therefore isotropic - form, but without flaws. (b) The second step will assume a domain structure such that each domain contains one or more aligned cracks. For lack of any better term, we call this picture the "random polycrystals of cracked-grains model." Both stages of the analysis use very similar tools, since bounds and estimates for polycrystals of pure crystalline grains are known, and then the cracked grains act in a very similar way - although possibly introducing a different crystal symmetry (i.e., hexagonal symmetry for aligned cracks in an isotropic background instead of the cubic symmetry for the metals considered). Nevertheless, the methods for all the pertinent symmetry groups are well-known and readily available for our use [11].

The second section presents the basic analysis for electrical (or, alternatively, thermal) 
conduction. The third section presents the corresponding analysis for the elastic properties. The fourth section then introduces the cross-property estimates and bounds for the crackedgrains system. The final section summarizes our results and conclusions.

\section{Conductivity/resistivity for random polycrystals of cracked grains}

\subsection{Uncorrelated random cracks}

Bristow [8] quotes work of Stroh [12] showing that the change $\Delta \rho$ in the resistivity $\rho$ of a medium containing a ribbon-shaped crack of width $2 a$ and length $\ell$ in a cube of unit volume is

$$
\frac{\Delta \rho}{\rho_{0}}=\frac{\pi a^{2} \ell}{3} .
$$

We will call this the "mixture theory" result, as it arises (and this fact will be shown explicitly later in the paper) by a simple average over the effects of the ribbon-shaped crack on resistivity along the plane of the crack (which produces two negligible contributions) and perpendicular to the crack face (which is the main effect). This model is a very simple one that makes no explicit assumptions concerning the local spatial correlations among the cracks, although nevertheless assuming implicitly that the crack density is low, and that the individual cracks are far from each other and, therefore, have negligible crack-crack interactions.

In general, for crystalline metallic materials, it is possible to have an anisotropic electrical conductivity (or resistivity) tensor associated with each metallic grain. This fact does not cause us any conceptual difficulties, and the details of finding an effective isotropic conductivity or resistivity tensor for the random polycrystal case are essentially the same as what we will be doing (in the next subsection) for the cracked grains. However, for some

crystal symmetries, such as those for the cubic metals that Bristow specifically considered, the electrical conductivity is inherently isotropic - so we actually have no work to do in this regard for such metals. TABLE 1 gives the values for the isotropic resistivity of the three metals $(\mathrm{Al}, \mathrm{Cu}, \mathrm{Ni})$ that Bristow considered, which are readily available in handbooks (or on the internet). 


\subsection{Random polycrystals of cracked grains: resistivity}

In order to have a rather precise model for comparison purposes, and to get a better feeling for just how much difference it makes whether we treat local order in the microstructure very accurately or not, we will now consider a model material called a "random polycrystal of cracked grains." Suppose we first construct a random polycrystal by packing small bits of a host material (assumed to be isotropic metallic crystallites - at the moment without any cracks present) into a large container in a way such that the axis of symmetry of these crystallites appears randomly over all possible orientations and also so that no misfit of surfaces (and therefore no misfit porosity between grains) is left in the resulting composite. This procedure produces statistically isotropic polycrystalline grains from the anisotropic metal crystallites. Next, at a more macroscopic level (called the mesoscopic scale), we introduce cracks into these statistically isotropic grains. Then we use the polycrystal analysis a second time to create the desired final model. (The concept and - therefore - the microgeometry is exactly the same for both electrical conductivity and the elastic properties.)

Figure 1 illustrates the type of microgeometry thus envisioned for the random polycrystals of cracked-grains model.

If the ratio of cracked grain size to the overall composite size is small enough so the usual implicit assumption of scale separation applies to this composite — but not so small that we are violating the continuum hypothesis - then we have an example of a random polycrystal of cracked grains. Both the transport properties and elastic behavior of the cracked grains themselves can be predicted using known methods, as we shall elaborate.

When studying conductivity $\left(\sigma=\rho^{-1}\right)$ in random polycrystals of cracked grains, it is most convenient to define a new canonical function (also see [13]):

$$
\Sigma_{X}(s)=\left[\frac{1}{3}\left(\frac{1}{\sigma_{\|}+2 s}+\frac{2}{\sigma_{\perp}+2 s}\right)\right]^{-1}-2 s,
$$

where $\sigma_{\|}$is the conductivity parallel to the axis of grain symmetry (i.e., perpendicular to the plane of the cracks in a particular grain) and $\sigma_{\perp}$ is the conductivity perpendicular to the axis of grain symmetry (and therefore parallel to the plane of the cracks) are the pertinent conductivities (on-axis and off-axis of symmetry, respectively) in each cracked grain. Then, the Hashin-Shtrikman bounds [10] for the conductivity of the random polycrystal are given 
by

$$
\sigma_{H S X}^{ \pm}=\Sigma_{X}\left(\sigma_{ \pm}\right)
$$

where $\sigma_{+}=\sigma_{\perp}$ and $\sigma_{-}=\sigma_{\|}$, when the cracks are air filled. In this case, the parameters $\sigma_{+}$and $\sigma_{-}$indicate the appropriate values for upper and lower bounds in (3), respectively. However, if the cracks happen to be filled with a highly conducting fluid, i.e., one that is more conducting than the surrounding grain material (this can easily happen for electrical conductivity in rocks, but not so easily for thermal conductivity in rocks, or for metals in general), then the subscripts \pm should be reversed in these expressions to indicate the appropriate significance of these bounds. These bounds are known not to be the most general ones since they rely on an implicit assumption that the grains are equiaxed. A more general lower bound that is known to be optimal is due to Schulgasser [14] and Avellaneda et al. $[15]:$

$$
\sigma_{A C L M X}^{-}=\Sigma_{X}\left(\sigma_{A C L M X}^{-} / 4\right)
$$

Helsing and Helte [16] have reviewed the state of the art for conductivity bounds and estimates $[17,18]$ for polycrystals, and in particular have noted that the self-consistent estimate [or CPA (i.e., coherent potential approximation)] for the random polycrystal conductivity is given by

$$
\sigma_{C P A X}^{*}=\Sigma_{X}\left(\sigma_{C P A X}^{*}\right)
$$

It is easy to show (5) always lies between the two rigorous bounds $\sigma_{A C L M X}^{-}$and $\sigma_{H S X}^{+}$, and also between $\sigma_{H S X}^{-}$and $\sigma_{H S X}^{+}$. Note that $\sigma_{A C L M X}^{-}$and $\sigma_{H S X}^{-}$cross when $\sigma_{\perp} / \sigma_{\|}=10$, with $\sigma_{A C L M X}^{-}$becoming the superior lower bound for $\sigma_{\perp} / \sigma_{\|}$contrast ratios greater than 10. For present purposes, resistivity in metals does not typically increase due to cracking by more than $5 \%$ or $6 \%$, so these extreme behaviors are beyond our current interests.

In order to have an explicit model of the cracked grains, we will assume that the cracks are either penny-shaped or ribbon-shaped. In either case, the approximation for very thin cracks will be taken for the contributions to $\sigma_{\perp}$. This approximation states that the electrical current is essentially undisturbed in both of these directions (parallel to the crack face), so

$$
\sigma_{\perp}=\sigma_{0}=\frac{1}{\rho_{0}} .
$$

If the cracks are ribbon-shaped, i.e., having a long dimension proportional to $\ell$ (where $\ell$ is actually defined as a length per unit volume) and a short dimension of length $2 a$, then 
- using the hydrodynamic analogy [19, 20] (i.e., for potential flow, the crack is analogous to a thin plate that oscillates in a fluid, and the added mass due to this oscillation is mathematically equivalent to the added resistance in the electrical problem) - we find easily that

$$
\frac{1}{\sigma_{\|}} \simeq \rho_{0}\left(1+\pi a^{2} \ell\right)
$$

The correction term (proportional to $\ell$ ) has the geometric significance of being the cylinder volume (per unit volume) of the entrained fluid of the ribbon-shaped plate oscillating broadside (i.e., perpendicular to face of the plate). This expression is approximate since we expect there to be a small correction due to the fact that the effects are not uniform within the cylinder volume in question. The corresponding result for a penny-shaped crack (or circular plate using the hydrodynamic analogy) includes an additional factor of $2 / \pi$ multiplying the spherical volume $4 \pi a^{3} / 3$, where $a$ is the radius of the crack face. Such correction factors are always in the range 0 to 1 , so (7) can be viewed as an upper bound on the actual value of the resistivity correction. However, these correction effects are usually subtle and small compared with the uncertainties in our knowledge of details of any real crack geometry, so this formula is therefore a reasonable, practical approximation for our current studies.

Stroh's result (1) is then easily recovered from the mixture theory average:

$$
\rho=\frac{1}{3}\left(\frac{1}{\sigma_{\|}}+\frac{2}{\sigma_{\perp}}\right) .
$$

Derivation of this formula assumes cracks are fairly uniformly and isotropically distributed in both space and angular orientation.

Examples of the predicted results from these formulas are displayed in Figure 2 for the metals considered by Bristow: aluminum, copper, and nickel.

\section{Elasticity for random polycrystals of cracked grains}

\subsection{Uncorrelated random cracks and the noninteraction approximation}

Again, for the elastic behavior of a system containing a set of ribbon-shaped cracks, Bristow [8] concludes that the effective bulk modulus $K^{*}$, shear modulus $G^{*}$, and Young's modulus $E^{*}$ have the following behaviors:

$$
\frac{K_{0}-K^{*}}{K^{*}}=-\frac{\Delta K}{K^{*}}=\frac{2 \pi a^{2} \ell}{3} \frac{\left(1-\nu_{0}^{2}\right)}{\left(1-2 \nu_{0}\right)}
$$




$$
\frac{G_{0}-G^{*}}{G^{*}}=-\frac{\Delta G}{G^{*}}=\frac{\pi a^{2} \ell}{15}\left(10-7 \nu_{0}\right)
$$

and

$$
\frac{E_{0}-E^{*}}{E^{*}}=-\frac{\Delta E}{E^{*}}=\frac{2 \pi a^{2} \ell}{15}\left(5-4 \nu_{0}\right)\left(1+\nu_{0}\right)
$$

These results are similar to, but not identical with, the noninteraction approximation as discussed by Zimmerman [21] for penny-shaped cracks. For comparison, Zimmerman's results are:

$$
\begin{gathered}
-\frac{\Delta K}{K^{*}}=\frac{16\left(1-\nu_{0}^{2}\right) \Gamma}{9\left(1-2 \nu_{0}\right)}, \\
-\frac{\Delta G}{G^{*}}=\frac{32\left(1-\nu_{0}\right)\left(5-\nu_{0}\right) \Gamma}{45\left(2-\nu_{0}\right)},
\end{gathered}
$$

and, since $3 / E=1 / 3 K+1 / G$, we also have

$$
-\frac{\Delta E}{E^{*}}=\frac{16\left(1-\nu_{0}^{2}\right)\left(10-3 \nu_{0}\right) \Gamma}{45\left(2-\nu_{0}\right)},
$$

where, for penny-shaped cracks having radius $a, \Gamma \equiv a^{3} N_{c}$ - with $N_{c}$ being the number of cracks per unit volume. Note that equations (9) and (12) for bulk modulus agree exactly if $\Gamma \equiv 3 \pi a^{2} \ell / 8$, while the factors involving $\nu_{0}$ in (10) and (13) for shear modulus and in (11) and (14) for Young's modulus do not agree in general - although for small values of $\nu_{0}$ $(\leq 0.5)$ their magnitudes are very close. Also note that $(1)$ can be rewritten as

$$
\frac{\Delta \rho}{\rho}=\frac{8}{9} \Gamma
$$

if we take $\Gamma \equiv 3 \pi a^{2} \ell / 8$ as the definition of $\Gamma$ for the ribbon-shaped cracks.

As mentioned previously, it is useful to imagine that there is first a random polycrystal of uncracked grains, and that somehow the cracks are introduced later into these grains. Of course, it is not likely that this is how real materials are formed, but it is handy to make this assumption for the sake of the two stage computation. The first stage gives us grains that are polycrystalline on a very fine scale. Then we can use standard methods [11] to compute the effect elastic constants of these uncracked grains. This process then results in the bounds and estimates illustrated in TABLE 2 .

\subsection{Crack-influence parameters}

Once we have the formulas discussed so far, we can then proceed to the second step for the elastic problem by introducing cracks into these already once homogenized grains. In order 
to justify this approach, we necessarily assume that the cracks are themselves mesoscopic (i.e., somewhat more macroscopic) compared to the many microcrystals that compose the effectively isotropic grains of our model. Thus, we are necessarily assuming a degree of scale separation between the metallic crystals composing the grains, and the cracks in the cracked-grains model.

Sayers and Kachanov [22] have shown that one convenient way to deduce the effective constants of an anisotropic cracked medium is first to deduce crack-influence parameters $\eta_{1}$ and $\eta_{2}$ from easily computed isotropic results, and then decompose the formulas into the desired results for the anisotropic situation.

The compliance correction matrix for simple isotropically distributed cracks - such as penny-shaped or ribbon-shaped cracks - takes the form:

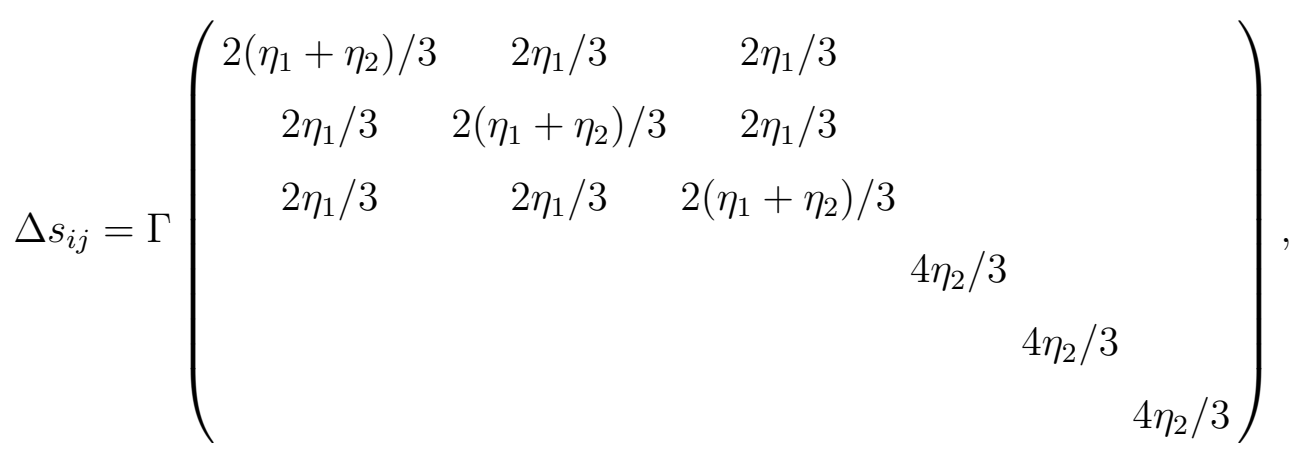

The known results appear in many places including [22-24]. We find in particular that

$$
\begin{gathered}
\frac{1}{G^{*}}-\frac{1}{G_{0}}=\frac{4 \eta_{2} \Gamma}{3}, \\
\frac{1}{K^{*}}-\frac{1}{K_{0}}=2\left(3 \eta_{1}+\eta_{2}\right) \Gamma,
\end{gathered}
$$

and

$$
\frac{1}{E^{*}}-\frac{1}{E_{0}}=\frac{2\left(\eta_{1}+\eta_{2}\right) \Gamma}{3} .
$$

These formulas can be solved for $\eta_{2}$ and $\eta_{1}$. The results are:

$$
\eta_{2} \Gamma=\frac{3}{4}\left(\frac{1}{G^{*}}-\frac{1}{G_{0}}\right)
$$

and

$$
\eta_{1} \Gamma=\frac{1}{6}\left(\frac{1}{K^{*}}-\frac{1}{K_{0}}\right)-\frac{1}{4}\left(\frac{1}{G^{*}}-\frac{1}{G_{0}}\right)=\frac{3}{2}\left(\frac{1}{E^{*}}-\frac{1}{E_{0}}\right)-\frac{3}{4}\left(\frac{1}{G^{*}}-\frac{1}{G_{0}}\right) .
$$

These values can then be related back to equations (9)-(11) for the ribbon-shaped cracks or to (12)-(14) for the penny-shaped cracks, as desired. 
Once these values of $\eta_{1}$ and $\eta_{2}$ have been deduced theoretically from the preceding formulas, then the compliance correction matrix for oriented (here we assume horizontal, i.e., $z$ - or $x_{3}$-axis of symmetry) cracks takes the form:

$$
\Delta s_{i j}=\Gamma\left(\begin{array}{cccccc}
0 & 0 & \eta_{1} & & & \\
0 & 0 & \eta_{1} & & \\
\eta_{1} & \eta_{1} & 2\left(\eta_{1}+\eta_{2}\right) & & \\
& & & 2 \eta_{2} & & \\
& & & 2 \eta_{2} & \\
& & & & 0
\end{array}\right)
$$

This result is the correction matrix used to deduce the perturbed values of the compliance matrix elements $s_{i j}^{*}=s_{i j}^{0}+\Delta s_{i j}$. There can also be higher order corrections (i.e., higher powers of $\Gamma$ ) associated with the compliance corrections [24], but these are related to the crack-crack interactions and we will not pursue these issues in the present work.

\subsection{Random polycrystals of cracked grains: elastic constants}

For the elastic properties of the cracked grains (having approximately hexagonal symmetry), the only explicit results needed in the following analysis for the polycrystals of cracked-grains model are the Reuss and Voigt averages for the grains themselves, which are $1 / K_{R}=2 s_{11}+2 s_{12}+4 s_{13}+s_{33}$ for the Reuss bulk modulus in terms of compliances ( $s_{i j}$ 's), or

$$
\frac{1}{K_{R}-c_{13}}=\frac{1}{c_{11}-c_{66}-c_{13}}+\frac{1}{c_{33}-c_{13}},
$$

in terms of stiffnesses $\left(c_{i j}\right.$ 's), and

$$
K_{V}=\left[2\left(c_{11}+c_{12}\right)+4 c_{13}+c_{33}\right] / 9
$$

for the Voigt average of bulk modulus. Similarly, the Voigt average for shear modulus of the stiffness matrix may be written as

$$
\mu_{V}=\frac{1}{5}\left(G_{\mathrm{eff}}^{v}+2 c_{44}+2 c_{66}\right)
$$

This expression can be taken as the definition of $G_{\mathrm{eff}}^{v}$. Eq. (25) implies that $G_{\mathrm{eff}}^{v}=\left(c_{11}+\right.$ $\left.c_{33}-2 c_{13}-c_{66}\right) / 3$. In fact, $G_{\text {eff }}^{v}$ is the energy per unit volume in a grain when a pure 
uniaxial shear strain of unit magnitude is applied to the grain along its axis of symmetry [25]. Similarly, the Reuss average for shear modulus is

$$
\mu_{R}=\left[\frac{1}{5}\left(\frac{1}{G_{\mathrm{eff}}^{r}}+\frac{2}{c_{44}}+\frac{2}{c_{66}}\right)\right]^{-1},
$$

which is also a rigorous lower bound on the overall shear modulus of the polycrystal [26]. $G_{\text {eff }}^{r}$ is the energy per unit volume in a grain when a pure uniaxial shear stress of unit magnitude is applied to the grain along its axis of symmetry [25]. Since each cracked grain has hexagonal symmetry (we assume that the cracks in each grain are all aligned with the same axis of symmetry), the product formulas $3 G_{\mathrm{eff}}^{r} K_{V}=3 G_{\mathrm{eff}}^{v} K_{R}=\omega_{+} \omega_{-} / 2=c_{33}\left(c_{11}-c_{66}\right)-c_{13}^{2}$ are valid [25]. The symbols $\omega_{ \pm}$stand for the quasi-compressional and quasi-uniaxial shear eigenvalues of each and every grain.

Once this notation has been established, it is then straightforward to express the Peselnick-Meister bounds on bulk modulus for polycrystals having grains with hexagonal symmetry [27] as

$$
K_{P M}^{ \pm}=\frac{K_{V}\left(G_{\mathrm{eff}}^{r}+Y_{ \pm}\right)}{\left(G_{\mathrm{eff}}^{v}+Y_{ \pm}\right)}
$$

where

$$
Y_{ \pm}=\frac{G_{ \pm}}{6}\left(\frac{9 K_{ \pm}+8 G_{ \pm}}{K_{ \pm}+2 G_{ \pm}}\right)=\Theta\left(K_{ \pm}, G_{ \pm}\right) .
$$

The precise values of the parameters $G_{ \pm}$and $K_{ \pm}$(being shear and bulk moduli of the HS isotropic comparison material) were given algorithmically by Watt and Peselnick [28]. Similarly,

$$
\frac{1}{\mu_{P M}^{ \pm}+Y_{ \pm}}=\frac{1}{5}\left[\frac{1-A_{ \pm}\left(K_{V}-K_{ \pm}\right)}{R_{ \pm}\left(K_{V}-K_{ \pm}\right)+G_{\mathrm{eff}}^{v}+Y_{ \pm}}+\frac{2}{c_{44}+Y_{ \pm}}+\frac{2}{c_{66}+Y_{ \pm}}\right]
$$

for the effective shear modulus $\mu^{*}$ of the polycrystal. The meaning of $Y_{ \pm}$is the same in (27) and (29). Here $A_{ \pm}=\frac{-1}{K_{ \pm}+4 G_{ \pm} / 3}, B_{ \pm}=\frac{2 A_{ \pm}}{15}-\frac{1}{5 G_{ \pm}}$, and $R_{ \pm}=A_{ \pm} / 2 B_{ \pm}$. These bounds are of Hashin-Shtrikman type, but were first obtained for hexagonal symmetry by Peselnick and Meister [27], with some corrections supplied later by Watt and Peselnick [28].

Since we now have analytical forms for the bounds in (27)-(29), it is possible to make the substitutions $K_{ \pm} \rightarrow K^{*}$ and $\mu_{ \pm} \rightarrow \mu^{*}$, as well as $K_{P M}^{ \pm} \rightarrow K^{*}$ and $\mu_{P M}^{ \pm} \rightarrow \mu^{*}$. Then, we arrive at a type of self-consistent (SC) estimate that will always lie between these rigorous bounds. Bounds and estimates for the three metals considered by Bristow [8] are presented in TABLE 3, where $G_{S C}=\mu^{*}, K_{S C}=K^{*}$, and $3 / E_{S C}=1 / 3 K_{S C}+1 / G_{S C}$ in making the connection between the notation in this subsection and that in the rest of the paper. 
Examples of the predicted results from these formulas are displayed in Figure 3.

\section{Cross-property Bounds and Estimates}

We combine the results of the previous two sections by producing crossplots of inverse elastic moduli versus resistivity for each of the same three metals considered by Bristow [8]: aluminum, copper, and nickel. The results are presented in Figure 4.

As shown already in Figures 2 and 3, the numerical results were computed for the crackdensity range $0.0 \leq \Gamma \leq 0.2$. This is a fairly large range for $\Gamma$, and so we do expect that some significant crack-crack interactions should start to appear for $\Gamma>0.05$. However, the calculations were nevertheless restricted for simplicity to the non-interaction approximation within each grain of the hypothetical polycrystal that we are studying. Some crack-crack interactions occur implicitly in the model due to the random domain structure of the polycrystal, but these effects are surely not as strong as crack-crack interactions would be if present within each grain - if we had allowed this to happen in the model (see Berryman and Grechka [24] for an example where crack-crack interactions were permitted in a very similar modeling situation).

We notice the following general characteristics of the plots in Figure 4. For aluminum, as the resistivity increases by about $5 \%$, the bulk modulus decreases by about $90 \%$ (i.e., a factor of 1.9) and the shear modulus by about 25\%. For copper, as the resistivity increases by about $5 \%$, the bulk modulus decreases by about $100 \%$ (i.e., a factor of 2 ) and the shear modulus by about $30 \%$. For nickel, as the resistivity increases by about $6 \%$, the bulk modulus decreases by about $90 \%$ (i.e., a factor of 1.9) and the shear modulus by about $30-35 \%$.

By way of comparison, Bristow's data for resistivity showed that the increase of resistivity for aluminum (going from soft to hard) was about 4\%, for copper about 4\%, and for nickel about $2 \%$. The change in static measurements for the shear modulus for the same materials was about $6 \%$ for aluminum, about $3 \%$ for copper, and about $3 \%$ for nickel; however, the sign of the change was opposite what would be expected from the simple theory of Bristow (and also from the theory presented here) for both copper and nickel. Furthermore, the magnitudes of these changes are all much smaller than what one might expect from the theory if the changes were really due to the introduction of more microcracks. This situation

led Bristow to conclude that the effects observed were not due to microcracks after all: so 
he says that "the hypothesis that on cold-working dislocation pile-up to form microcracks in metals is not correct."

We should also point out however that a more complete analysis than the one given here will surely show that, when crack-crack interactions are introduced into the grains of the polycrystal model as presented, it will happen that the effects of the cracks overall on the elastic moduli can be much smaller than that predicted without the crack-crack interaction. For example, two cases are studied by Berryman and Grechka [24]. The first example has Poisson's ratio $\nu_{0}=0.00$. It was found for this extreme case that as $\Gamma$ ranged from 0.0 to 0.2 , the noninteraction approximation for the bulk modulus predicted about a $36 \%$ maximum decrease in the bulk modulus, while simulations showed that the actual maximum decrease while taking crack-crack interactions into account was only about 27\%; the results for the shear modulus were similar, giving about $38 \%$ maximum decrease for noninteraction, and $28 \%$ maximum decrease when crack-crack interactions were included. The second example studied was for Poisson's ratio $\nu_{0}=0.4375$. In this case, the observed decrease in the bulk modulus for the noninteraction approximation ranged up to about $230 \%$ (i.e., a factor of 3.3), while the maximum decrease was only about $110 \%$ (i.e., a factor of 2.1) for simulations including crack-crack interactions at $\Gamma \simeq 0.2$. The results differed between the cases $\nu_{0}=0.4375$ and $\nu_{0}=0.0$ in one possibly significant way which is that the presence of the crack-crack interactions also seemed to have a larger effect at small $\Gamma$ for the $\nu_{0}=0.4375$ case than it did for the case $\nu_{0}=0.00$. So reduction of modulus changes due to crack-crack interactions may be more significant in general for materials with higher Poisson's ratios, and especially so at low crack densities. Since the metals considered have reasonably high Poisson's ratios, the importance of crack-crack interactions needs to be studied more carefully.

\section{Summary and Conclusions}

Our results ultimately agree qualitatively with those of Bristow, i.e., confirming that microcracking is not likely to be the main cause of the observed elastic behavior of coldworked metals. However, it also becomes clear that the mixture theory approach to the analysis is too simple and that crack-crack interactions are necessary for proper quantitative study of Bristow's problem. The analysis of such crack-crack interactions is beyond our 
current scope and, therefore, extensions of the analysis in that direction will be pursued in future work. These issues are however nontrivial as has been demonstrated already by various researchers $[29,30]$.

\section{Acknowledgments}

Work performed under the auspices of the U.S. Department of Energy, at the Lawrence Berkeley National Laboratory under Contract No. DE-AC03-76SF00098. Support was provided specifically by the Geosciences Research Program of the DOE Office of Basic Energy Sciences, Division of Chemical Sciences, Geosciences and Biosciences.

\section{REFERENCES}

[1] J. G. Berryman. G. W. Milton, Microgeometry of random composites and porous media, J. Phys. D: Appl. Phys. 21 (1988) 87-94.

[2] L. M. Schwartz, N. Martys, D. P. Bentz, E. J. Garboczi, S. Torquato, Cross-property relations and permeability estimation in model porous media, Phys. Rev. E 64 (1993) 4584-4591.

[3] M. Kachanov, I. Sevostianov, Explicit cross-property correlations for porous materials with anisotropic microstructures, J. Mech. Phys. Solids 49 (1) (2001) 1-25.

[4] G. W. Milton, The Theory of Composites, Cambridge University Press, Cambridge, UK, 2002.

[5] I. Sevostianov, M. Kachanov, Explicit cross-property correlations for anisotropic twophase composite materials, J. Mech. Phys. Solids 50 (2) (2002) 253-282.

[6] S. Torquato, Random Heterogeneous Materials: Microstructure and Macroscopic Properties, Springer, New York, 2002, pp. 422-423, 598.

[7] S. Torquato, A. Donev, A. G. Evans, C. J. Brinker, Manufacturable extremal lowdielectric, high stiffness porous materials, J. Appl. Phys. 97 (12) (2005) 124103.

[8] J. Bristow, Microcracks, and the static and dynamic elastic constants of annealed and heavily cold-worked metals, British Journal of Applied Physics 11 (1960) 81-85. 
[9] Z. Hashin, S. Shtrikman, A variational approach to the theory of the elastic behaviour of polycrystals, J. Mech. Phys. Solids 10 (1962) 343-352.

[10] Z. Hashin, S. Shtrikman, Conductivity of polycrystals, Phys. Rev. 130 (1963) 129-133.

[11] J. G. Berryman, Bounds and self-consistent estimates for elastic constants of random polycrystals with hexagonal, trigonal, and tetragonal symmetries, J. Mech. Phys. Solids 53 (10) (2005) 2141-2173.

[12] A. N. Stroh, The existence of microcracks after cold work, Phil. Mag. 2 (13) (1957) 1-4.

[13] J. G. Berryman, Measures of microstructure to improve estimates and bounds on elastic constants and transport coefficients in heterogeneous media, Mech. Mat. 38 (8-10) (2006) $732-747$.

[14] K. Schulgasser, Sphere assemblage model for polycrystals and symmetric materials, J. Appl. Phys. 54 (1983) 1380-1382.

[15] M. Avellaneda, A. V. Cherkaev, K. A. Lurie, G. W. Milton, On the effective conductivity of polycrystals and a three-dimensional phase-interchange inequality, J. Appl. Phys. 63 (1988) 4989-5003.

[16] J. Helsing, A. Helte, Effective conductivity of aggregates of anisotropic grains, J. Appl. Phys. 69 (1991) 3583-3588.

[17] D. A. G. Bruggeman, Berechnung verschiedener physikalischer Konstanten von heterogenen Substanzen, Ann. Physik. (Leipzig) 24 (1935) 636-679.

[18] R. Landauer, The electrical resistance of binary metallic mixtures, J. Appl. Phys. 23 (1952) 779-784.

[19] J. G. Berryman, Effective conductivity by fluid analogy for a porous insulator filled with a conductor, Phys. Rev. B 27 (1983) 7789-7792.

[20] H. Lamb, Hydrodynamics, Cambridge University Press, Cambridge, UK, 1993, pp. 152156.

[21] R. W. Zimmerman, Compressiblity of Sandstones, Elsevier, Amsterdam, 1991, p. 123. 
[22] C. M. Sayers, M. Kachanov, A simple technique for finding effective elastic constants of cracked solids for arbitrary carck orientation statistics, Int. J. Solids Struct. 27 (1991) $671-680$.

[23] Z. P. Bažant, J. Planas, Fracture and Size Effect in Concrete and Other Quasibrittle Materials, CRC Press, New York, 1998, pp. 560-563.

[24] J. G. Berryman, V. Grechka, Random polycrystals of grains containing cracks: Model of quasistatic elastic behavior for fractured systems, J. Appl. Phys. 100 (2006) 113527.

[25] J. G. Berryman, Poroelastic shear modulus dependence on pore-fluid properties arising in a model of thin isotropic layers, Geophys. J. Int. 127 (2004) 415-425.

[26] R. Hill, Elastic properties of reinforced solids: Some theoretical principles, Proc. Phys. Soc. London A 65 (1952) 349-354.

[27] L. Peselnick, R. Meister, Variational method of determining effective moduli of polycrystals: (A) Hexagonal symmetry, (B) trigonal symmetry, J. Appl. Phys. 36 (1965) 2879-2884.

[28] J. P. Watt, L. Peselnick, Clarification of the Hashin-Shtrikman bounds on the effective elastic moduli of polycrystals with hexagonal, trigonal, and tetragonal symmetries, J. Appl. Phys. 51 (1980) 1525-1531.

[29] O. S. Kruger, E. H. Saenger, S. J. Oates, S. A. Shapiro, A numerical study on reflection coefficients of fractured media, Geophysics 72 (4) (2007) D61-D67.

[30] V. Grechka, Comparison of the non-inteaction and differential schemes in predicting the effective elastic properties of fractured media, Int. J. Fracture 144 (3) (2007) 181-188.

[31] C. D. Hodgman, R. C. Weast, R. S. Shankland, S.M. Selby, Handbook of Chemisty and Physics, The Chemical Rubber Publishing Co., The Chemical Rubber Publishing Co., 1962, pp. 2665-2668.

[32] M. J. P. Musgrave, Crystal Acoustics: Introduction to the Study of Elastic Waves and Vibrations in Crystals, Acoustical Society of America, New York, 2003, pp. 278-279. 
TABLE 1. Isotropic electrical resistivity values $\rho$ at $20^{\circ} \mathrm{C}$ for three metals having cubic symmetry: aluminum, copper, and nickel. Values are all taken from Hodgman et al. [31].

\begin{tabular}{|c|c|c|c|}
\hline \hline Resistivity & $\mathrm{Al}$ & $\mathrm{Cu}$ & $\mathrm{Ni}$ \\
\hline$\rho(\Omega \cdot \mathrm{m})$ & $2.828 \times 10^{-8}$ & $1.724 \times 10^{-8}$ & $6.993 \times 10^{-8}$ \\
\hline \hline
\end{tabular}


TABLE 2. Cubic crystal stiffness constants (in GPa) for three metals: aluminum, copper, and nickel. Values are all taken from Musgrave [32]. These values are the ones used as input to determine the effective isotropic values quoted in TABLE 3.

\begin{tabular}{|c|c|c|c|}
\hline \hline Material & $c_{11}^{*}$ & $c_{12}^{*}$ & $c_{44}^{*}$ \\
\hline $\mathrm{Al}$ & 108.2 & 61.3 & 28.5 \\
$\mathrm{Cu}$ & 168.4 & 121.4 & 75.4 \\
$\mathrm{Ni}$ & 246.5 & 147.3 & 124.7 \\
\hline \hline
\end{tabular}


TABLE 3. Estimated elastic stiffness constants (all values in GPa except for Poisson's ratios which are dimensionless) for polycystalline aggregates of three metals: aluminum, copper, and nickel. Subscripts correspond respectively to Voigt (V), Reuss (R), and Hashin-Shtrikman (HS) bounds, while the self-consistent estimates for polycrystals are labeled SC. Constants evaluated are effective isotropic bulk modulus $(K)$, shear modulus $(G)$, Young's modulus $(E)$, and Poisson's ratio $(\nu)$.

\begin{tabular}{|c|c|c|c|}
\hline \hline Constant & $\mathrm{Al}$ & $\mathrm{Cu}$ & $\mathrm{Ni}$ \\
\hline$K_{V}=K_{R}$ & 76.9 & 137.1 & 180.4 \\
$G_{V}$ & 26.5 & 54.6 & 94.7 \\
$G_{H S}^{+}$ & 26.4 & 49.4 & 88.1 \\
$G_{S C}$ & 26.4 & 48.2 & 86.9 \\
$G_{H S}^{-}$ & 26.4 & 46.0 & 84.9 \\
$G_{R}$ & 26.2 & 40.0 & 77.7 \\
$E_{S C}$ & 71.0 & 129.4 & 224.6 \\
$\nu_{S C}$ & 0.346 & 0.343 & 0.292 \\
\hline \hline
\end{tabular}


TABLE 4. Crack-influence parameters $\eta_{1}$ and $\eta_{2}$ for the metals considered in the text: aluminum, copper, and nickel. Values used for the metallic elastic constants are the SC values from TABLE 3 . In all three cases, note that $\left|\eta_{1}\right| / \eta_{2} \simeq 0.03$.

\begin{tabular}{|c|c|c|c|}
\hline \hline Crack Parameters & $\mathrm{Al}$ & $\mathrm{Cu}$ & $\mathrm{Ni}$ \\
\hline$\eta_{1}\left(\mathrm{GPa}^{-1}\right)$ & -0.0013 & -0.0007 & -0.0004 \\
$\eta_{2}\left(\mathrm{GPa}^{-1}\right)$ & 0.0372 & 0.0204 & 0.0120 \\
\hline \hline
\end{tabular}




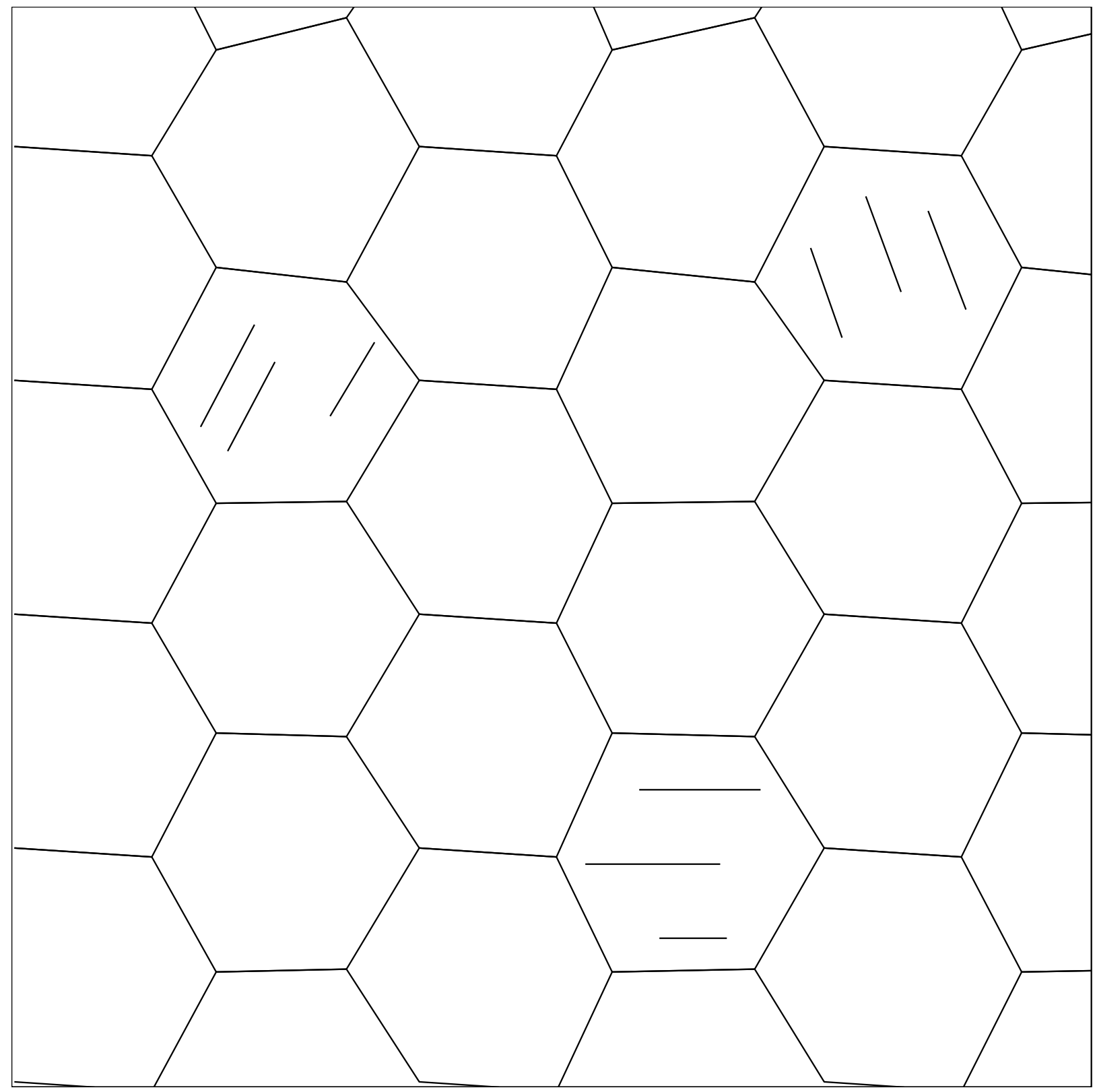

FIG. 1: Schematic illustrating the random polycrystals of cracked-grains model. Grains are assumed to fit tightly so there is no misfit porosity, although there is a very small amount of porosity due to the presence of the cracks themselves. The shapes of the grains are not necessarily the same, and the symmetry axes of the grains (three examples shown) are randomly oriented so the overall polycrystal is equiaxed (statistically isotropic). 
(a)

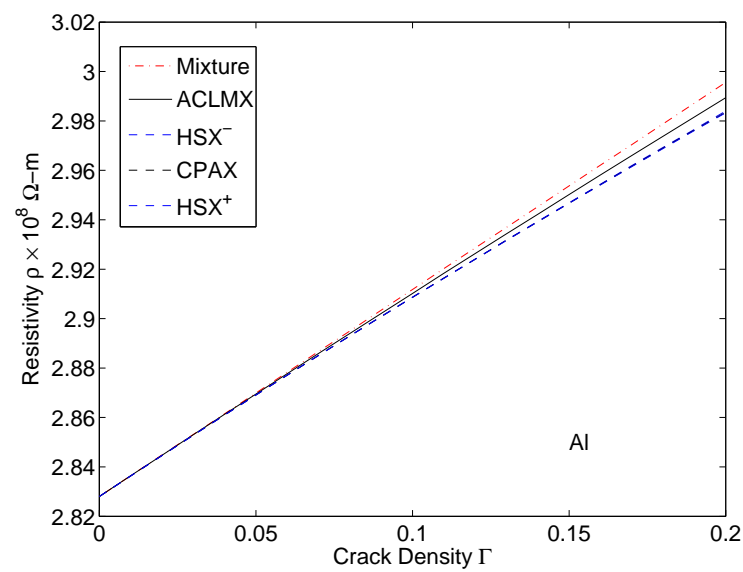

(b)

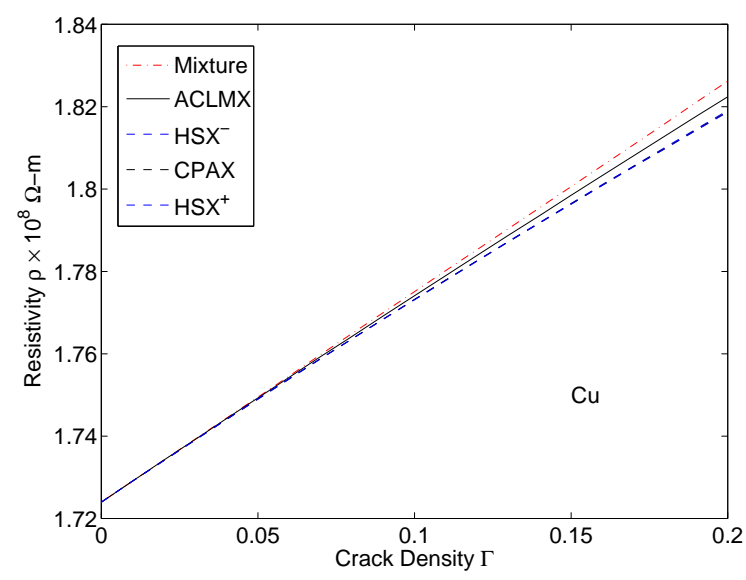

(c)

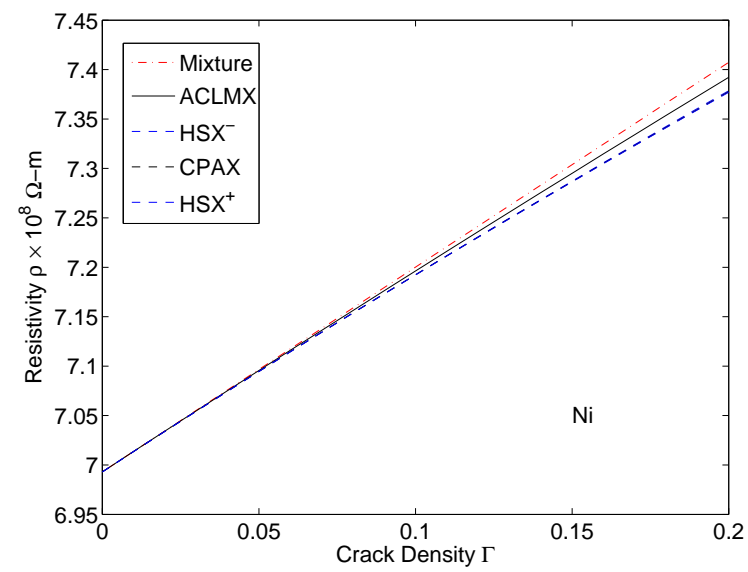

FIG. 2: Examples of resistivity calculations for the polycrystals of cracked-grains model for three metals: aluminum, copper, and nickel. The CPA effective medium theory results always lie between the two Hashin-Shtrikman bounds. The mixture theory result is always out of range, being above the ACLMX upper bound on resistivity for this model. 
(a)

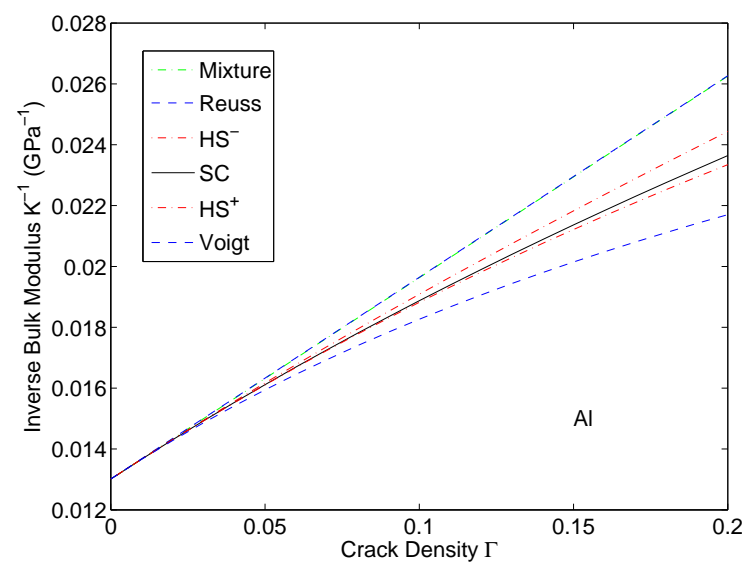

(c)

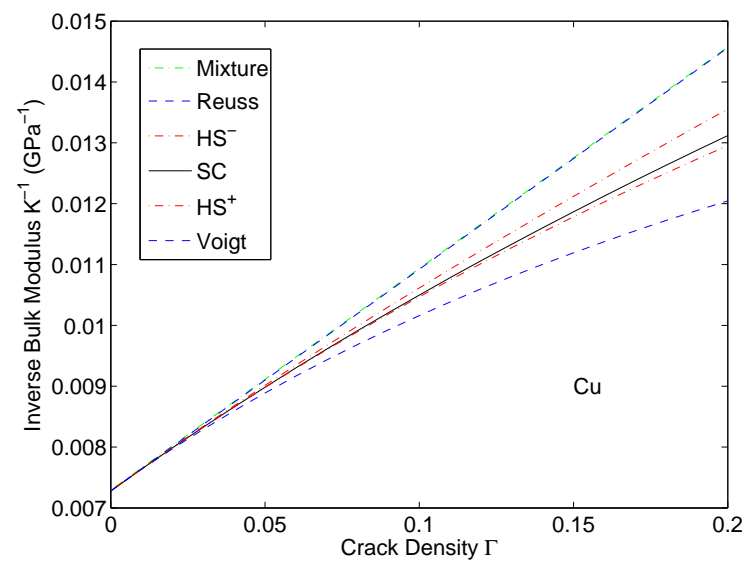

(e)

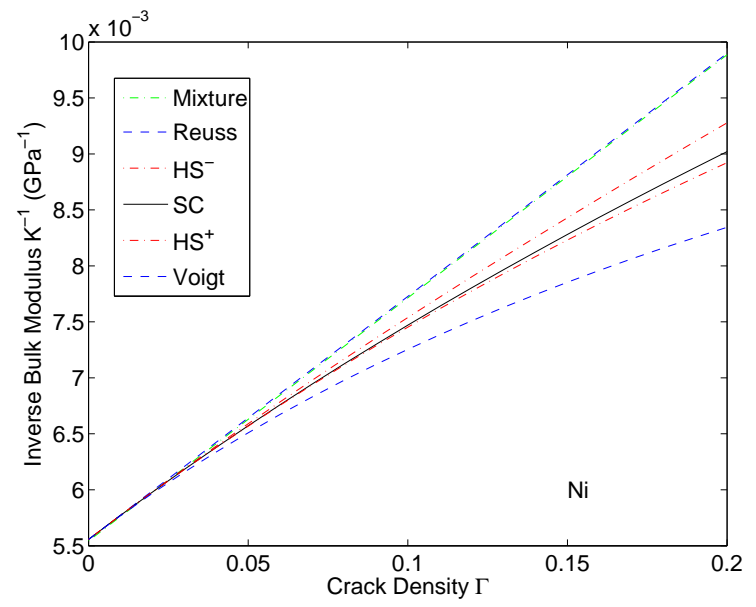

(b)

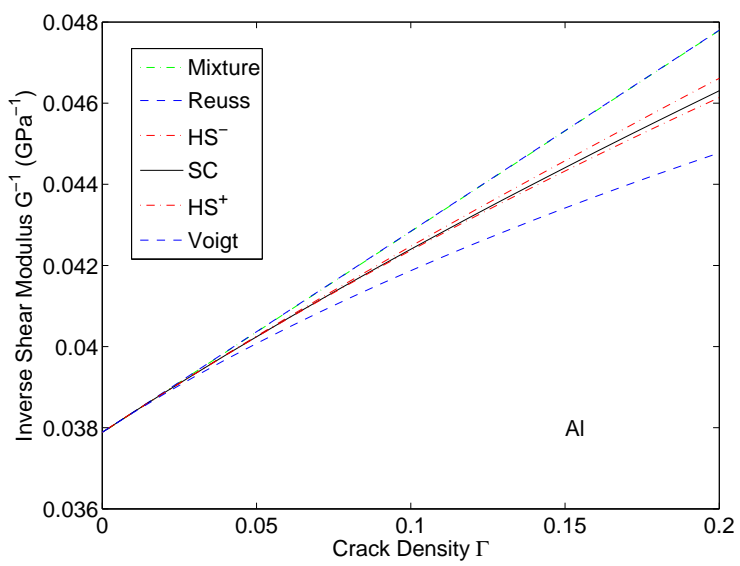

(d)

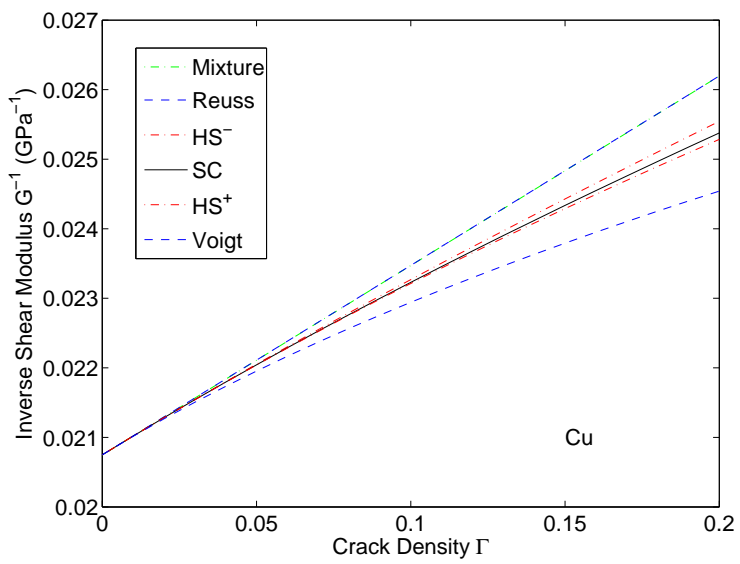

(f)

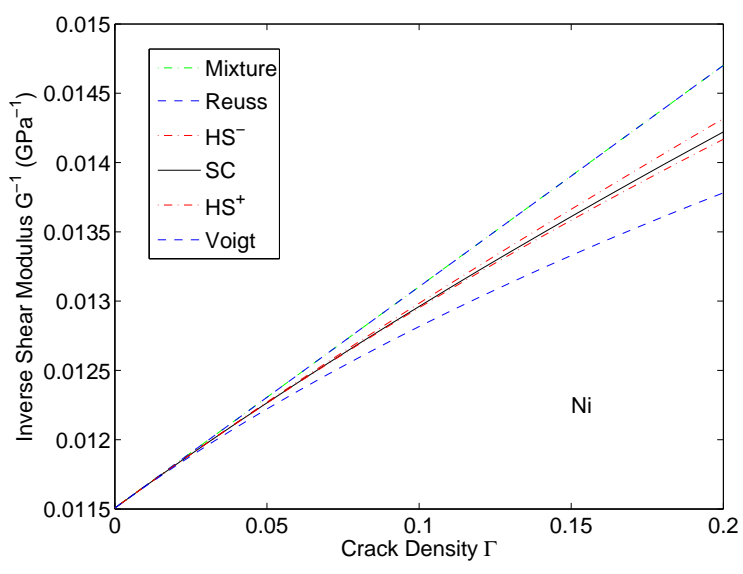

FIG. 3: Examples of compliance calculations (inverse bulk modulus and inverse shear modulus) for the polycrystals of cracked-grains model for aluminum (a,b), copper $(\mathrm{c}, \mathrm{d})$, and nickel $(\mathrm{e}, \mathrm{f})$. The SC effective medium theory results always lie between the two Hashin-Shtrikman bounds. The mixture theory result (which is also the same as the noninteraction approximation) turns out to be the same as the Reuss average for this model, since we have not included any crack-crack interaction within the grains in the current analysis. 
(a)

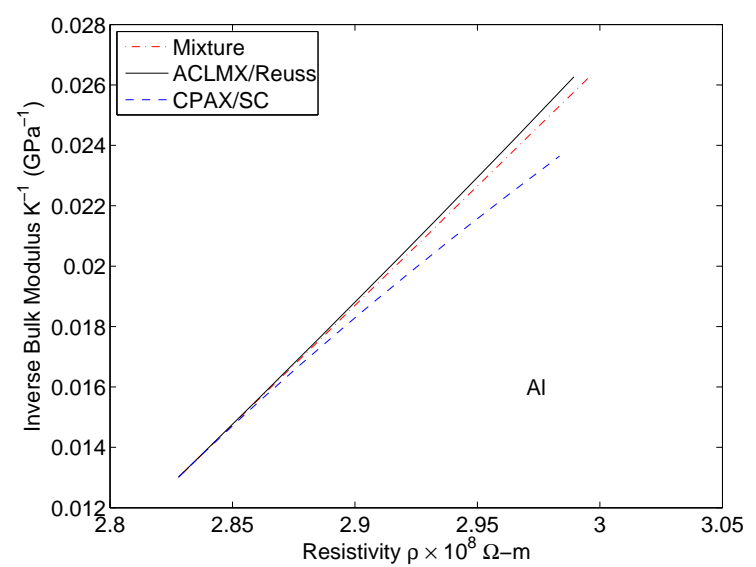

(c)

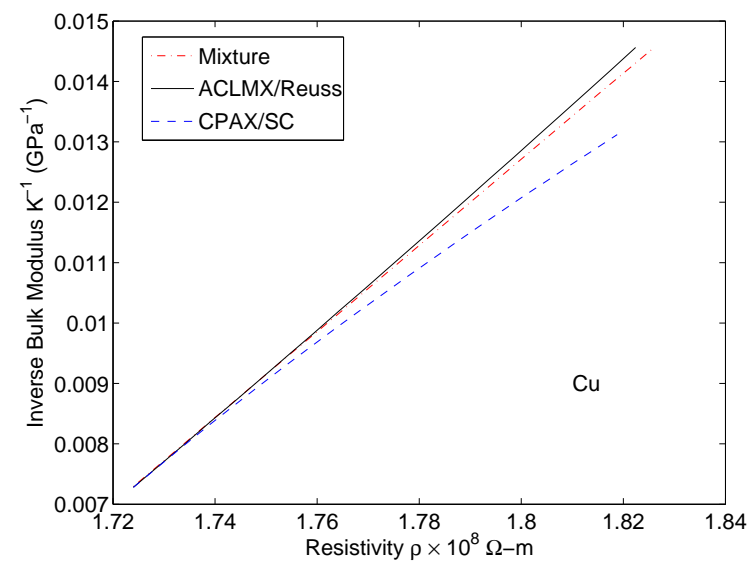

(e)

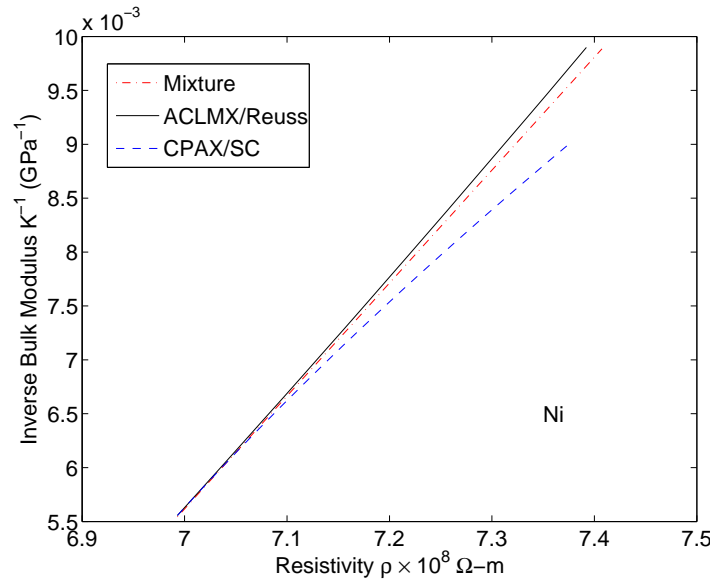

(b)

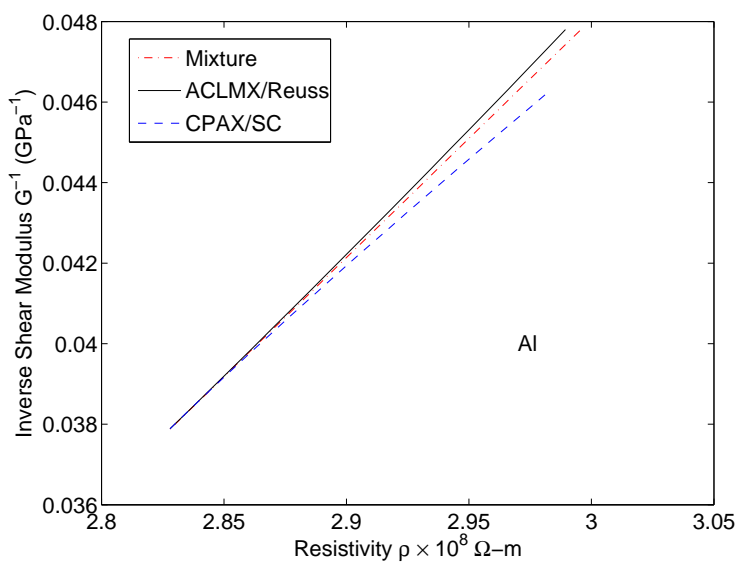

(d)
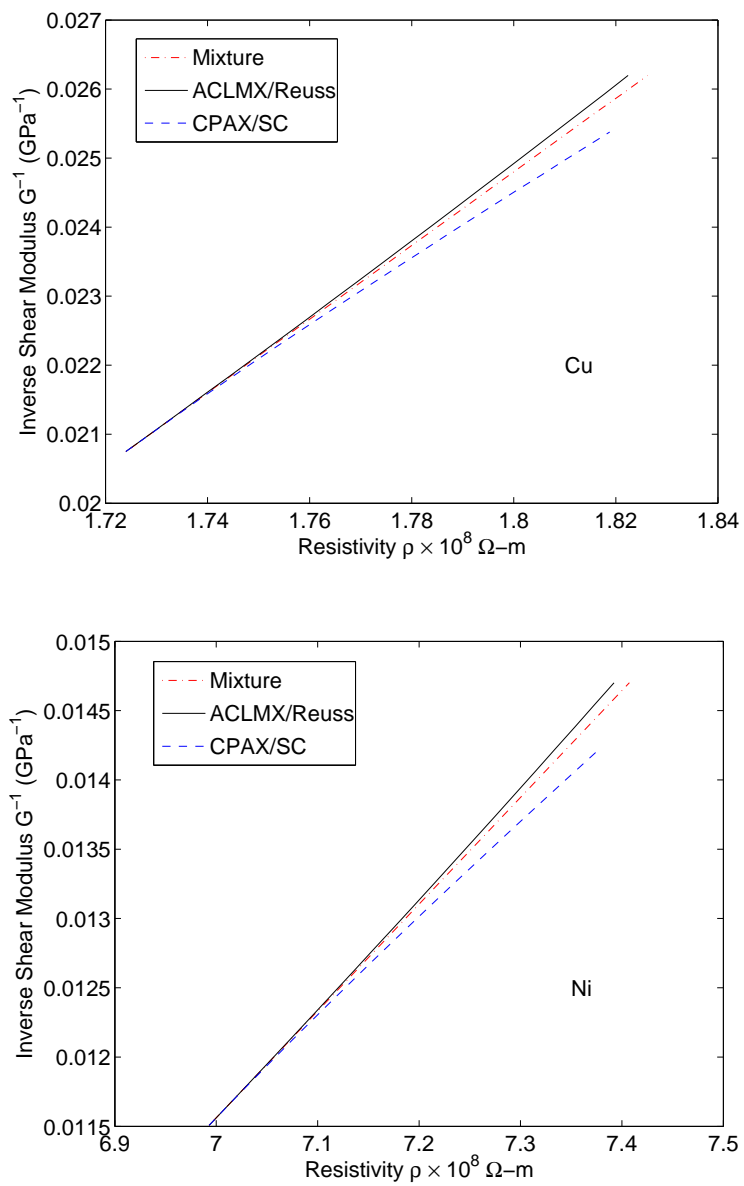

FIG. 4: Examples of cross-property plots for the three metals considered: aluminum (a,b), copper $(\mathrm{c}, \mathrm{d})$, and nickel $(\mathrm{e}, \mathrm{f})$. The SC effective medium results for inverse bulk and shear moduli are plotted versus the CPAX results for resisitivity. The Reuss bounds for bulk and shear moduli are plotted versus the ACLMX bounding results for the resistivity. The mixture theory results (which are also the same as the noninteraction approximation) for inverse bulk and shear moduli are plotted against the mixture theory results for resistivity. 